\title{
A SHAPE OPTIMAL CONTROL PROBLEM WITH CHANGING SIGN DATA*
}

\author{
GIUSEPPE BUTTAZZO ${ }^{\dagger}$ AND BOZHIDAR VELICHKOV
}

\begin{abstract}
In this paper we consider a shape optimization problem in which the data in the cost functional and in the state equation may change sign, and so no monotonicity assumption is satisfied. Nevertheless, we are able to prove that an optimal domain exists. We also deduce some necessary conditions of optimality for the optimal domain. The results are applied to show the existence of an optimal domain in the case where the cost functional is completely identified, while the right-hand side in the state equation is only known up to a probability $P$ in the space $L^{2}(D)$.
\end{abstract}

Key words. shape optimization, free boundary, capacitary measures, stochastic optimization

AMS subject classifications. 49Q10, 49J45, 49A22, 35J25, 49B60

DOI. $10.1137 / 17 \mathrm{M} 1126564$

1. Introduction. In this paper we consider a shape optimization problem of the form

$$
\min \{F(\Omega): \Omega \in \mathcal{A}\},
$$

where $F$ is the shape cost function and $\mathcal{A}$ is the class of admissible domains. For this kind of problem in general one should not expect the existence of an optimal domain, since minimizing sequences could be made of finely perforated domains, leading at the limit to existence of only relaxed solutions that are not domains but Borel measures. In some particular cases, however, an optimal domain exists; the most general existence result providing optimal solutions that are domains and not measures is still given by Theorem 2.5 of [6] (see also [5]), where the crucial assumption is that the shape cost functional $F$ is monotone decreasing with respect to the set inclusion. A similar result for monotone costs in the framework of optimization problems for Schrödinger potentials has been obtained in [7].

The cost functional $F$ we consider here is not in general monotone decreasing for the set inclusion; nevertheless we are able to prove the existence of an optimal domain for it. We fix

- a bounded Lipschitz domain $D \subset \mathbb{R}^{d}$,

- a right-hand side $f \in L^{2}(D)$, and

- a cost coefficient $g \in L^{2}(D)$,

and we consider the admissible class of domains

$$
\mathcal{A}=\{\Omega \subset D, \Omega \text { quasi open, }|\Omega| \leq 1\}
$$

\footnotetext{
*Received by the editors April 21, 2017; accepted for publication (in revised form) March 13, 2018; published electronically May 10, 2018.

http://www.siam.org/journals/sima/50-3/M112656.html

Funding: The work of the first author was part of the project 2015PA5MP7 Calcolo delle Variazioni funded by the Italian Ministry of Research and University. The first author is a member of the Gruppo Nazionale per l'Analisi Matematica, la Probabilità e le loro Applicazioni (GNAMPA) of the Istituto Nazionale di Alta Matematica (INdAM). The work of the second author was partially supported by the LabEx PERSYVAL-Lab (ANR-11-LABX-0025-01) project GeoSpec and the project ANR COMEDIC.

$\dagger^{\dagger}$ Dipartimento di Matematica, Università di Pisa, 56127 Pisa, Italy (buttazzo@dm.unipi.it, http: //www.dm.unipi.it/pages/buttazzo/).

${ }^{\ddagger}$ Laboratoire Jean Kuntzmann (LJK), Université Grenoble Alpes, Bâtiment IMAG, 38401 SaintMartin-d'Hères, France (bozhidar.velichkov@univ-grenoble-alpes.fr, http://www.velichkov.it).
} 
where $|\cdot|$ denotes the Lebesgue measure in $\mathbb{R}^{d}$. In order that the problem be nontrivial we assume that $|D|>1$.

1.1. Statement of the problem and main results. For every $\Omega \in \mathcal{A}$ we denote by $u_{\Omega}$ the unique solution of the Dirichlet problem

$$
-\Delta u=f \quad \text { in } \quad \Omega, \quad u \in H_{0}^{1}(\Omega),
$$

where $H_{0}^{1}(\Omega)$ is the Sobolev space of functions in $H^{1}\left(\mathbb{R}^{d}\right)$ that vanish capacity quasi everywhere outside $\Omega$. The optimization problem we are dealing with is

$$
\min \left\{\int_{D} g(x) u_{\Omega}(x) d x: \Omega \in \mathcal{A}\right\}
$$

Note that, by the definition of $u_{\Omega}$, problem (1.3) is an optimal control problem, where $H_{0}^{1}(D)$ is the space of states, $\mathcal{A}$ is the set of controls, (1.2) is the state equation, and $\int_{D} g(x) u_{\Omega}(x) d x$ is the cost function. We stress the fact that we do not assume any sign condition on the data $f, g$.

It is well known that in the special case $g=-f / 2$ the optimization problem (1.3) can be written, through an Euler-Lagrange derivation and an integration by parts, as

$$
\min \{\mathcal{E}(\Omega): \Omega \in \mathcal{A}\}
$$

where $\mathcal{E}(\Omega)$ is the Dirichlet energy

$$
\mathcal{E}(\Omega)=\min \left\{\int\left[\frac{1}{2}|\nabla u|^{2}-f(x) u\right] d x: u \in H_{0}^{1}(\Omega)\right\} .
$$

This would allow us to see easily, thanks to the inclusion of the Sobolev spaces

$$
\Omega_{1} \subset \Omega_{2} \Longrightarrow H_{0}^{1}\left(\Omega_{1}\right) \subset H_{0}^{1}\left(\Omega_{2}\right),
$$

that the shape function $\mathcal{E}(\Omega)$ is decreasing with respect to the set inclusion, and then an immediate application of the existence theorem Theorem 2.5 of [6] would give a solution $\Omega_{o p t}$ of problem (1.3), with the additional property that $\left|\Omega_{\text {opt }}\right|=1$.

The same conclusion would easily hold when $f \geq 0$ and $g \leq 0$; indeed, in this case, thanks to the maximum principle, the solutions $u_{\Omega}$ would be monotonically increasing with respect to $\Omega$, and again the shape cost function $\Omega \mapsto \int_{D} g(x) u_{\Omega}(x) d x$ would turn out to be decreasing with respect to $\Omega$, providing then (again by the existence theorem Theorem 2.5 of [6]) an optimal solution $\Omega_{o p t}$ of problem (1.3), with $\left|\Omega_{o p t}\right|=1$.

On the contrary, when $f$ and $g$ are general functions in $L^{2}(D)$, the existence theorem Theorem 2.5 of [6] cannot be applied and the existence of an optimal domain for the minimization problem (1.3) requires a deeper investigation. Our main existence result is the following.

Theorem 1.1. Let $f, g \in L^{2}(D)$ be given; then the minimization problem (1.3) admits a solution $\Omega_{\text {opt }}$ in the admissible class $\mathcal{A}$.

Moreover, the optimal sets have the following properties:

- if $g \geq 0$, we have either $\left|\Omega_{o p t}\right|=1$ or $\left|\Omega_{\text {opt }}\right|<1$ and $\{f<0\} \subset \Omega_{o p t}$ (Theorem 4.5); similarly, if $f \geq 0$, we have either $\left|\Omega_{\text {opt }}\right|=1$ or $\left|\Omega_{\text {opt }}\right|<1$ and $\{g<0\} \subset \Omega_{o p t}$; 
- if $\Omega_{o p t}$ is smooth, the state functions $u$ and $v$ on $\Omega_{o p t}$, corresponding to the solutions of the PDE (1.2) with right-hand side $f$ and $g$, respectively, satisfy

$$
\frac{\partial u}{\partial n} \frac{\partial v}{\partial n}=\text { const } \quad \text { on } \quad \partial \Omega_{o p t} \cap D,
$$

the constant being zero if $\left|\Omega_{\text {opt }}\right|<1$ (section 3);

- if $\left|\Omega_{o p t}\right|<1$ and $f \geq 0$, then the function $v_{\Omega}$, corresponding to the function $g$, is a solution of an obstacle problem (Proposition 5.4), and thus, under some appropriate assumptions on the regularity of $g$, the optimal set $\Omega_{o p t}$ is open and its boundary is smooth (Corollary 5.5);

- if $D=\mathbb{R}^{d}$ and $f, g$ are radially symmetric functions, $f$ radially decreasing and $g$ radially increasing, then the optimal set $\Omega_{o p t}$ is a ball centered in zero (Proposition 6.1).

1.2. A stochastic optimal control problem. A probabilistic counterpart of the optimization problem (1.3) is given by the case when the function $g$ appearing in the cost functional (1.3) is completely known, while the right-hand side $f$ in (1.2) has the form $f=f_{0}+h$, where $f_{0}$ is given and $h$ is some random perturbation. The purpose of such a model is to obtain shapes corresponding to mechanical structures that are robust and reliable even if the data are not completely known. Several models involving uncertainties have already been studied; from the numerical point of view we refer, for instance, to [1] and the references therein, while in most of the cases there are no available theoretical results, even in some simplified situations.

An interesting result in this spirit is concerned with the existence of optimal domains for the worst-case functional

$$
\min _{\Omega \in \mathcal{A}} \sup _{h \in L^{p}} \int_{D} g(x) R_{\Omega}\left(f_{0}+h\right) d x
$$

and was proved in [2] under the assumptions that $g \leq 0, f_{0}>0$, and the perturbation $h$ is small. Here $R_{\Omega}$ denotes the resolvent operator which associates to every $f \in L^{2}(D)$ the solution $u_{\Omega}$ of $(1.2)$.

Another situation of practical interest is when the perturbation $h$ belongs to some probability space and the cost functional is given by the average over all possible choices of $h$. The existence of a minimizer in this situation can be deduced from Theorem 1.1 without any smallness assumption on the uncertainty $h$.

More precisely, given a probability $P$ on $L^{2}(D)$, we aim to minimize the averaged cost

$$
F(\Omega)=\int\left(\int_{D} g(x) R_{\Omega}(f) d x\right) d P(f)
$$

over the admissible class $\mathcal{A}$ given by (1.1). We assume that the barycenter

$$
B_{P}:=\int f d P(f)
$$

belongs to $L^{2}(D)$ and we notice that it is well defined when $P$ has a finite first moment

$$
\int\|f\|_{L^{2}} d P(f)<+\infty .
$$


Thus, setting $\langle u, v\rangle:=\int_{D} u(x) v(x) d x$, for $u, v \in L^{2}(D)$, and using the fact that the resolvent operator $R_{\Omega}$ is self-adjoint, we obtain that the cost functional in (1.4) can be written as

$$
\begin{aligned}
F(\Omega) & =\int\left\langle R_{\Omega}(f), g\right\rangle d P(f)=\int\left\langle R_{\Omega}(g), f\right\rangle d P(f) \\
& =\left\langle R_{\Omega}(g), B_{P}(f)\right\rangle=\int_{D} g(x) R_{\Omega}\left(B_{P}\right) d x .
\end{aligned}
$$

In this way we are then in the framework of the existence result Theorem 1.1.

1.3. Organization of the paper. In section 3 we prove the existence of an optimal domain $\Omega_{\text {opt }}$ (Theorem 1.1). Then we compute the so-called shape derivative assuming that $\Omega_{o p t}$ is regular enough. The study of the regularity properties of the optimal domains is an interesting and difficult issue; obtaining the regularity of a general solution $\Omega_{o p t}$ from its minimality would be a very interesting result.

In section 4 we study the minimizers for which the constraint $\left|\Omega_{\text {opt }}\right| \leq 1$ is not saturated. Note that this is a rather general situation, since no monotonicity of the shape cost function is assumed. Nevertheless, in several cases $(f \geq 0$ and $\mid\{g<$ $0\} \mid \geq 1$ ) we may still obtain that the optimal domain verifies $\left|\Omega_{o p t}\right|=1$ as we see in Theorem 4.5. In section 5 we show that $\Omega_{o p t}$ is a solution of an obstacle problem, and as a consequence we obtain that it has a regular free boundary in the sense of Corollary 5.5.

Finally, in section 6 we study the case of radially symmetric functions $f$ and $g$. It is natural to expect that under this assumption the optimal domains are balls centered at zero. Also in this case the lack of monotonicity of the functional represents a difficult issue since the energy does not necessarily decrease under symmetrization. Nevertheless, we are able to prove that for every $\Omega$ there is a ball $B$ (not necessarily of the same measure as $\Omega$ ) having a smaller energy. We also provide an example of an optimal set $\Omega_{o p t}$ of measure strictly smaller than one.

2. Sobolev spaces, quasi open sets, and capacitary measures. In this section we briefly recall several notions related to capacity theory, quasi open sets, and capacitary measures; we refer the reader to the book [4] for more details concerning these notions.

2.1. Sobolev functions and their representatives. The Sobolev space $H^{1}\left(\mathbb{R}^{d}\right)$ is the closure of $C_{c}^{\infty}\left(\mathbb{R}^{d}\right)$ with respect to the norm

$$
\|u\|_{H^{1}}=\left(\int_{\mathbb{R}^{d}}|\nabla u|^{2} d x+\int_{\mathbb{R}^{d}} u^{2} d x\right)^{1 / 2} .
$$

For every function $u \in H^{1}\left(\mathbb{R}^{d}\right)$ there is a set $E_{u} \subset \mathbb{R}^{d}$ such that

- every point in $\mathbb{R}^{d} \backslash E_{u}$ is a Lebesgue point for $u$, that is,

$$
u\left(x_{0}\right)=\lim _{r \rightarrow 0} \frac{1}{\left|B_{r}\right|} \int_{B_{r}\left(x_{0}\right)} u(x) d x, \quad \text { for every } \quad x_{0} \in \mathbb{R}^{d} \backslash E_{u} ;
$$

- $E_{u}$ has capacity zero, $\operatorname{cap}\left(E_{u}\right)=0$, where for a set $E \subset \mathbb{R}^{d}, \operatorname{cap}(E)$ is defined as

$$
\operatorname{cap}(E):=\inf \left\{\|\phi\|_{H^{1}}^{2}: \phi \in H^{1}\left(\mathbb{R}^{d}\right), \phi=1 \text { in a neighborhood of } E\right\} .
$$

We notice that a Sobolev function $u$ is defined up to a set of zero capacity; that is, $u_{1} \sim u_{2}$ if and only if $\operatorname{cap}\left(\left\{u_{1} \neq u_{2}\right\}\right)=0$. 
2.2. Quasi open sets and the space $\boldsymbol{H}_{0}^{\mathbf{1}}(\Omega)$. We say that a set $\Omega \subset \mathbb{R}^{d}$ is quasi open if it is of the form $\Omega=\{u>0\}$ for some $u \in H^{1}\left(\mathbb{R}^{d}\right)$. We notice that all the open sets are quasi open. Given a quasi open set $\Omega \subset \mathbb{R}^{d}$ we define the Sobolev space

$$
H_{0}^{1}(\Omega):=\left\{u \in H^{1}\left(\mathbb{R}^{d}\right): \operatorname{cap}(\{u \neq 0\} \backslash \Omega)=0\right\} .
$$

We notice that $H_{0}^{1}(\Omega)$ is a closed subspace of $H^{1}\left(\mathbb{R}^{d}\right)$. In fact, if $u_{n} \rightarrow u$ in $H^{1}\left(\mathbb{R}^{d}\right)$, then up to a subsequence $u_{n} \rightarrow u$ pointwise outside of a set of zero capacity. If $\Omega$ is open, then $H_{0}^{1}(\Omega)$ coincides with the usual Sobolev space defined as the closure of $C_{c}^{\infty}(\Omega)$ with respect to the $H^{1}$ norm. Let $\Omega \subset \mathbb{R}^{d}$ be a quasi open set of finite measure, and let $f \in L^{2}(\Omega)$. We say that a function $u \in H_{0}^{1}(\Omega)$ is a solution of the equation

$$
-\Delta u=f \quad \text { in } \Omega, \quad u \in H_{0}^{1}(\Omega),
$$

if we have

$$
\int_{\Omega} \nabla u \cdot \nabla \varphi d x=\int_{\Omega} f \varphi d x \text { for every } \varphi \in H_{0}^{1}(\Omega) .
$$

2.3. Capacitary measures. We say that a nonnegative Borel measure $\mu$ is capacitary if for every set $E \subset \mathbb{R}^{d}$ with $\operatorname{cap}(E)=0$, we have $\mu(E)=0$. We denote by $\mathcal{M}_{\text {cap }}\left(\mathbb{R}^{d}\right)$ the class of capacitary measures on $\mathbb{R}^{d}$. In particular, if two functions $u_{1}$ and $u_{2}$ are in the same equivalence class of $H^{1}\left(\mathbb{R}^{d}\right)$, and $\mu$ is a capacitary measure, then $u_{1}$ and $u_{2}$ are in the same equivalence class of $L^{2}(\mu)$. For a quasi-open set $\Omega \subset \mathbb{R}^{d}$ and for a measure $\mu \in \mathcal{M}_{\text {cap }}\left(\mathbb{R}^{d}\right)$ we define the space

$$
H_{\mu}^{1}(\Omega)=H_{0}^{1}(\Omega) \cap L^{2}(\mu)=\left\{u \in H_{0}^{1}(\Omega): \int_{\mathbb{R}^{d}} u^{2} d \mu<\infty\right\} .
$$

For a given function $f \in L^{2}(\Omega)$ we say that $u \in H_{\mu}^{1}(\Omega)$ is a solution of the equation

$$
-\Delta u+\mu u=f \quad \text { in } \Omega, \quad u \in H_{\mu}^{1}(\Omega),
$$

if we have

$$
\int_{\Omega} \nabla u \cdot \nabla \varphi d x+\int_{\Omega} u \varphi d \mu=\int_{\Omega} f \varphi d x \quad \forall \varphi \in H_{\mu}^{1}(\Omega) .
$$

Let $\mu$ be a capacitary measure in $\mathbb{R}^{d}$. The set of finiteness $\Omega_{\mu}$ of $\mu$ is defined as

$$
\Omega_{\mu}=\bigcup_{u \in H_{\mu}^{1}\left(\mathbb{R}^{d}\right)}\{u \neq 0\}
$$

We notice that the set $\Omega_{\mu}$ is a quasi open set due to the fact that $H_{\mu}^{1}$ is separable. Moreover, if $\mu=0$ on $\Omega_{\mu}$, then $H_{\mu}^{1}\left(\mathbb{R}^{d}\right)=H_{0}^{1}\left(\Omega_{\mu}\right)$.

2.4. Convergence of capacitary measures. Consider a bounded open set $D \subset \mathbb{R}^{d}$ and the family of capacitary measures

$$
\mathcal{M}_{\text {cap }}(D)=\left\{\mu \in \mathcal{M}_{\text {cap }}\left(\mathbb{R}^{d}\right): \Omega_{\mu} \subset D\right\} .
$$

For every capacitary measure $\mu \in \mathcal{M}_{\text {cap }}(D)$ we consider the torsion function $w_{\mu}$, solution of the equation

$$
-\Delta w_{\mu}+\mu w_{\mu}=1 \quad \text { in } D, \quad w_{\mu} \in H_{\mu}^{1}(D)
$$


We notice that $w_{\mu}$ uniquely determines the measure $\mu$. In fact, we have

$$
\Omega_{\mu}=\left\{w_{\mu}>0\right\} \quad \text { and } \quad \mu=\frac{\Delta w_{\mu}+1}{w_{\mu}} \quad \text { on } \Omega_{\mu} .
$$

The set $\mathcal{M}_{\text {cap }}(D)$, endowed with the distance

$$
d_{\gamma}\left(\mu_{1}, \mu_{2}\right)=\left\|w_{\mu_{1}}-w_{\mu_{2}}\right\|_{L^{2}},
$$

is a compact metric space (see, for instance, [11]). Moreover, the family of capacitary measures $I_{\Omega}$ associated to smooth domains $\Omega \subset D$ is dense in $\mathcal{M}_{\text {cap }}(D)$, where the measure $I_{\Omega}$ is defined by

$$
I_{\Omega}(E)= \begin{cases}0 & \text { if } \operatorname{cap}(E \backslash \Omega)=0 \\ +\infty & \text { otherwise }\end{cases}
$$

3. Existence of optimal shapes and optimality conditions. In this section we prove the existence result Theorem 1.1. We first relax the problem to the class of capacitary measures $\mathcal{M}_{\text {cap }}(D)$ that represents the closure of the admissible class $\mathcal{A}$ with respect to the $\gamma$-convergence. The relaxed problem is written again as an optimal control problem, with admissible class given by

$$
\mathcal{M}=\left\{\mu \in \mathcal{M}_{\text {cap }}(D),\left|\Omega_{\mu}\right| \leq 1\right\},
$$

$\Omega_{\mu}$ being the set of finiteness of $\mu$. For every admissible $\mu \in \mathcal{M}$ we consider the state equation

$$
-\Delta u+\mu u=f \quad \text { in } \quad D, \quad u \in H_{0}^{1}(D) \cap L^{2}(\mu),
$$

and we indicate its unique solution by $u_{\mu}$. The relaxed optimization problem related to (1.3) can then be stated as

$$
\min \left\{\int_{D} g(x) u_{\mu} d x: \mu \in \mathcal{M}\right\}
$$

It is convenient to introduce the resolvent operator $R_{\mu}: L^{2}(D) \rightarrow L^{2}(D)$ which associates to every $f \in L^{2}(D)$ the solution $u_{\mu}$ of (3.1). Thanks to the fact that $R_{\mu}$ is self-adjoint, we can write the cost function as

$$
\int_{D} g(x) R_{\mu}(f) d x=\int_{D} R_{\mu}(g) f(x) d x .
$$

Proof of Theorem 1.1. It is well known that the relaxed admissible class $\mathcal{M}$ is compact with respect to $\gamma$-convergence and that the cost function is $\gamma$-continuous (see, for instance, [4]); therefore, an optimal relaxed solution $\mu$ to problem (3.2) exists.

For every bounded continuous function $\phi$ and for every $\varepsilon>0$ small enough we consider the capacitary measure $\mu_{\varepsilon}=(1+\varepsilon \phi) \mu$; since $\phi$ is bounded and $\varepsilon$ is small, we have that $\mu_{\varepsilon} \in \mathcal{M}$ and $\Omega_{\mu_{\varepsilon}}=\Omega_{\mu}$. Moreover, the spaces $H_{\mu_{\varepsilon}}^{1}$ and $H_{\mu}^{1}$ coincide. Let us denote by $u_{\varepsilon}$ the solution of the PDE

$$
-\Delta u_{\varepsilon}+\mu_{\varepsilon} u_{\varepsilon}=f \quad \text { in } D, \quad u_{\varepsilon} \in H_{\mu_{\varepsilon}}^{1}
$$


and by $u$ the solution of

$$
-\Delta u+\mu u=f \quad \text { in } D, \quad u \in H_{\mu}^{1} .
$$

By the minimality of $\mu$ we have

$$
\int_{D} g u_{\varepsilon} d x \geq \int_{D} g u d x
$$

which gives

$$
\int_{D} g \frac{u_{\varepsilon}-u}{\varepsilon} d x \geq 0
$$

Denoting by $w_{\varepsilon}$ the function $\left(u_{\varepsilon}-u\right) / \varepsilon$, we have that $w_{\varepsilon}$ satisfies the PDE

$$
-\Delta w_{\varepsilon}+\mu w_{\varepsilon}=-\phi u_{\varepsilon} \mu \quad \text { in } D, \quad w_{\varepsilon} \in H_{\mu}^{1} .
$$

Since $\mu_{\varepsilon} \gamma$-converges to $\mu$ we have that $u_{\varepsilon} \rightarrow u$ weakly in $H_{\mu}^{1}$; hence $w_{\varepsilon} \rightarrow w$ weakly in $H_{\mu}^{1}$, where $w$ is the solution of the PDE

$$
-\Delta w+\mu w=-\phi u \mu \quad \text { in } D, \quad w \in H_{\mu}^{1} .
$$

Passing to the limit in (3.3) as $\varepsilon \rightarrow 0$ gives

$$
0 \leq \int_{D} g w d x=\int_{D} g R_{\mu}(-\phi u \mu) d x=-\int_{D} R_{\mu}(g) \phi u d \mu .
$$

Since $\phi$ is arbitrary, we obtain that

$$
R_{\mu}(g) u=0, \quad \mu \text {-a.e. }
$$

Since $u=0$ where $\mu=+\infty$, by the form of the cost functional, without loss of generality we may assume that $\Omega_{\mu}=\{u \neq 0\}$. Analogously, since the cost functional can also be written as $\int_{D} R_{\mu}(g) f d x$, we may assume that $\mu=+\infty$ on $R_{\mu}(g)=0$. Thus by (3.4) the capacitary measure $\mu$ takes only values 0 and $+\infty$, and hence it is a domain.

We now formally deduce the optimality condition on the boundary of an optimal set $\Omega \subset D$ (for the rigorous proof we refer the reader to [14, Chapter 5]). We assume that $\Omega$ is sufficiently regular $\left(\partial \Omega \in C^{2, \alpha}\right.$ ), and we set for simplicity $u=R_{\Omega}(f)$ and $v=R_{\Omega}(g)$. For a smooth vector field $V \in C_{c}^{\infty}\left(D ; \mathbb{R}^{d}\right)$ we consider the perturbation $\Omega_{t}=(I d+t V)(\Omega)$ and the solutions $u_{t}=R_{\Omega_{t}}(f)$ and $v_{t}=R_{\Omega_{t}}(g)$. The formal derivatives

$$
u^{\prime}=\left.\frac{d}{d t}\right|_{t=0} u_{t} \quad \text { and } \quad v^{\prime}=\left.\frac{d}{d t}\right|_{t=0} v_{t}
$$

are solutions, respectively, of the problems

$$
\begin{array}{llll}
\Delta u=0 & \text { in } \Omega, & u^{\prime}+V \cdot \nabla u=0 & \text { on } \partial \Omega, \\
\Delta v=0 & \text { in } \Omega, & v^{\prime}+V \cdot \nabla v=0 & \text { on } \partial \Omega .
\end{array}
$$

Thus, the derivative of the cost functional is given by

$$
\begin{aligned}
\left.\frac{d}{d t}\right|_{t=0} \int_{\Omega_{t}} u_{t} g d x & =\int_{\Omega} u^{\prime} g d x=\int_{\Omega} \nabla u^{\prime} \nabla v d x-\int_{\partial \Omega} u^{\prime} \frac{\partial v}{\partial n} \\
& =\int_{\partial \Omega} \frac{\partial v}{\partial n} V \cdot \nabla u=\int_{\partial \Omega} V \cdot n \frac{\partial u}{\partial n} \frac{\partial v}{\partial n} .
\end{aligned}
$$


We now consider two cases.

Case 1. If the volume constraint is saturated, that is, $|\Omega|=1$, then we have to consider perturbations only with respect to divergence-free vector fields $V$. In this case we obtain

$$
\int_{\partial \Omega}(V \cdot n) \frac{\partial u}{\partial n} \frac{\partial v}{\partial n}=0 \quad \text { for every } V \in C_{c}^{\infty}\left(D ; \mathbb{R}^{d}\right) \text { such that } \operatorname{div} V=0,
$$

which gives the optimality condition

$$
\frac{\partial u}{\partial n} \frac{\partial v}{\partial n}=\text { const } \quad \text { on } \partial \Omega
$$

Case 2. If the volume constraint is not saturated, that is, $|\Omega|<1$, then we have

$$
\int_{\partial \Omega} V \cdot n \frac{\partial u}{\partial n} \frac{\partial v}{\partial n}=0 \quad \text { for every } V \in C_{c}^{\infty}\left(D ; \mathbb{R}^{d}\right)
$$

which gives the optimality condition

$$
\frac{\partial u}{\partial n} \frac{\partial v}{\partial n}=0 \quad \text { on } \partial \Omega
$$

In the case when $f \geq 0$, we have that $|\nabla u|>0$ on the boundary of the optimal set $\Omega=\{u>0\}$. Thus the optimality condition can be written in the simplified form

$$
\frac{\partial v}{\partial n}=0 \quad \text { on } \partial \Omega
$$

This situation is untypical for the shape optimization problem, where the cost functional is usually monotone with respect to the set inclusion. We give an explicit example of a case when the constraint is not saturated in section 6 . In the next section we analyze this type of solution and its connection with the obstacle problem.

4. Minimizers with nonsaturated constraint. In this section we consider minimizers $\Omega$ which do not saturate the volume constraint, that is, $|\Omega|<1$. We restrict our attention to the case $f \geq 0$ on $D$, while the cost coefficient $g$ may change sign. Equivalently, since the resolvent operators are self-adjoint, we may consider $g \geq 0$ and $f$ changing sign. In Theorem 4.5 we prove that an optimal set $\Omega$ necessarily contains the set $\{g<0\}$. In subsection 5 we establish a relation of the minimizer $\Omega$ with the obstacle problem.

The main result of this section is a necessary condition of optimality (Theorem 4.5). The argument is carried out from the point of view of the state function $u=$ $R_{\Omega}(f)$ relative to a nonnegative right-hand side $f$. Before we pass to the statement and the proof of Theorem 4.5 we recall several classical results concerning the function $u$.

Remark 4.1. Let $f \in L^{2}(D)$, and let $u \in H_{0}^{1}(D)$ be a nonnegative function such that $\Delta u+f \geq 0$ on $D$ in the sense of distributions; that is,

$$
\int_{D}(-\nabla u \cdot \nabla \varphi+f \varphi) d x \geq 0 \quad \text { for every nonnegative } \varphi \in C_{c}^{\infty}(D) .
$$

It is well known that $\nu:=\Delta u+f$ is a (positive) measure. Moreover, $\Delta u+f$ is a Radon measure in $D$. In fact, if $B_{r}\left(x_{0}\right) \subset D$, there is a nonnegative function $\varphi \in C_{c}^{\infty}(D)$ such that $\varphi=1$ on $B_{r}\left(x_{0}\right)$; thus

$$
(\Delta u+f)\left(B_{r}\left(x_{0}\right)\right) \leq \int_{D} \varphi d \nu=\int_{D}(-\nabla u \cdot \nabla \varphi+f \varphi) d x<+\infty .
$$


In what follows we use an important characterization of $\Delta u+f$ to construct competitors for the solution of problem (3.2). For the proof we refer the reader to [12] (Theorem 5.1).

Lemma 4.2. Let $f \in L^{2}(D)$, and let $u \in H_{0}^{1}(D)$ be a nonnegative function. Then the following conditions are equivalent:

(i) $\Delta u+f \mathbb{1}_{\{u>0\}} \geq 0$ on $D$ in the sense of distributions;

(ii) there exists a capacitary measure $\mu \in \mathcal{M}_{\text {cap }}(D)$ such that $\Omega_{\mu}=\{u>0\}$ and

$$
-\Delta u+\mu u=f \quad \text { in } D, \quad u \in H_{\mu}^{1} .
$$
of

Let now $\Omega \subset \mathbb{R}^{d}$ be a bounded quasi open set, and let $u \in H_{0}^{1}(\Omega)$ be the solution

$$
-\Delta u=f \quad \text { in } \Omega, \quad u \in H_{0}^{1}(\Omega) .
$$

The following lemma describes the behavior of $u$ around the boundary points of low density for $\Omega$. The result is classical and we give the proof for the sake of completeness.

Lemma 4.3. Let $r_{0}>0, x_{0} \in \mathbb{R}^{d}$, and $f \in L^{2}\left(B_{r_{0}}\left(x_{0}\right)\right)$, with $f \geq 0$. Suppose that

$$
M:=\sup _{0<r \leq r_{0}}\left(\frac{1}{\left|B_{r}\right|} \int_{B_{r}\left(x_{0}\right)} f^{2}(x) d x\right)^{1 / 2}<+\infty .
$$

Then there exists a constant $\varepsilon>0$, depending only on the dimension $d$ and on $M$, such that if $\Omega$ satisfies the hypothesis

$$
\frac{\left|B_{r}\left(x_{0}\right) \cap \Omega\right|}{\left|B_{r}\right|} \leq \varepsilon \quad \text { for every } 0<r<r_{0},
$$

then for the solution $u$ of (4.1) we have the estimate

$$
\frac{1}{r^{2}\left|B_{r}\right|} \int_{B_{r}\left(x_{0}\right)}|\nabla u|^{2} d x \leq 2^{d+2} \sup \left\{1, \frac{1}{r_{0}^{2}\left|B_{r_{0}}\right|} \int_{B_{r_{0}}\left(x_{0}\right)}|\nabla u|^{2} d x\right\} .
$$

Proof. Suppose, without loss of generality, that $x_{0}=0$. Let $0<r<r_{0}$ and $\phi \in C_{0}^{\infty}\left(B_{r}\right)$ be a function such that $0 \leq \phi \leq 1$ on $B_{r}, \phi=1$ on $B_{r / 2}$, and $|\nabla \phi| \leq 3 / r$. The proof is obtained by iteration of the following Caccioppoli inequality:

$$
\begin{aligned}
\int_{B_{r / 2}}|\nabla u|^{2} d x & \leq \int_{B_{r}}|\nabla(\phi u)|^{2} d x=\int_{B_{r}}|\nabla \phi|^{2} u^{2} d x+\int_{B_{r}} \nabla u \cdot \nabla\left(\phi^{2} u\right) d x \\
& =\int_{B_{r}}|\nabla \phi|^{2} u^{2} d x+\int_{B_{r}} f \phi^{2} u d x \\
& \leq \frac{9}{r^{2}} \int_{B_{r}} u^{2} d x+\left(\int_{B_{r}} f^{2} d x\right)^{1 / 2}\left(\int_{B_{r}} u^{2} d x\right)^{1 / 2} .
\end{aligned}
$$

Now, there are constants $\Lambda_{1}>0$ and $\delta_{0}>0$ such that if $\left|\Omega \cap B_{r}\right| \leq \delta_{0}\left|B_{r}\right|$ and $v \in H^{1}\left(B_{r}\right)$ is such that $v=0$ on $B_{r} \backslash \Omega$, then

$$
\int_{B_{r}} v^{2} d x \leq \Lambda_{1} r^{2}\left(\frac{\left|\Omega \cap B_{r}\right|}{\left|B_{r}\right|}\right)^{2 / d} \int_{B_{r}}|\nabla v|^{2} d x .
$$


This follows by a rescaling of the estimate from Lemma 4.4 applied to the domain $D=B_{1}$. Thus, we obtain

$$
\int_{B_{r / 2}}|\nabla u|^{2} d x \leq 9 \Lambda_{1} \varepsilon^{2 / d} \int_{B_{r}}|\nabla u|^{2} d x+\left(\int_{B_{r}} f^{2} d x\right)^{1 / 2}\left(\Lambda_{1} \varepsilon^{2 / d} r^{2} \int_{B_{r}}|\nabla u|^{2} d x\right)^{1 / 2} .
$$

Dividing by $r^{2}\left|B_{r}\right|$, we get

$$
\begin{aligned}
\frac{1}{r^{2}\left|B_{r}\right|} \int_{B_{r / 2}}|\nabla u|^{2} d x & \leq \frac{9 \Lambda_{1} \varepsilon^{2 / d}}{r^{2}\left|B_{r}\right|} \int_{B_{r}}|\nabla u|^{2} d x+\left(\frac{1}{\left|B_{r}\right|} \int_{B_{r}} f^{2} d x\right)^{1 / 2}\left(\frac{\Lambda_{1} \varepsilon^{2 / d}}{r^{2}\left|B_{r}\right|} \int_{B_{r}}|\nabla u|^{2} d x\right)^{1 / 2} \\
& \leq \frac{9 \Lambda_{1} \varepsilon^{2 / d}}{r^{2}\left|B_{r}\right|} \int_{B_{r}}|\nabla u|^{2} d x+M \Lambda_{1}^{1 / 2} \varepsilon^{1 / d}\left(\frac{1}{r^{2}\left|B_{r}\right|} \int_{B_{r}}|\nabla u|^{2} d x\right)^{1 / 2} .
\end{aligned}
$$

Let us indicate by $r_{n}$ and $a_{n}$ the quantities

$$
r_{n}=r_{0} 2^{-n}, \quad a_{n}=\frac{1}{r_{n}^{2}\left|B_{r_{n}}\right|} \int_{B_{r_{n}}}|\nabla u|^{2} d x .
$$

Then, for $\varepsilon$ small enough we have

$$
a_{n+1} \leq \frac{1}{2} a_{n}+\frac{1}{2} a_{n}^{1 / 2}
$$

which gives that $a_{n} \leq \sup \left\{1, a_{0}\right\}$, for every $n \geq 1$.

In the next lemma we prove the estimate (4.2), which essentially follows by the argument explained in Example 5.3 of [8], carried out in dimension 2, but which can be adapted to any dimension $d \geq 2$.

Lemma 4.4. Let $D \subset \mathbb{R}^{d}$ be a connected bounded open set with Lipschitz boundary. Then, there are constants $\Lambda_{1}>0$ and $\delta_{0}>0$ such that, for every $v \in H^{1}(D)$ for which $|\{v \neq 0\}| \leq \delta_{0}$, we have

$$
\int_{D} v^{2} d x \leq \Lambda_{1}|\{v \neq 0\}|^{2 / d} \int_{D}|\nabla v|^{2} d x
$$

Proof. We first notice that by replacing $v$ by $|v|$ we can assume that $v \geq 0$ in $D$. We then notice that since $D$ is Lipschitz and connected, there are constants $\delta_{0}$ and $C_{D}$ such that the following isoperimetric inequality holds in $D$ :

$$
|\Omega|^{\frac{d-1}{d}} \leq C_{D} P(\Omega ; D) \quad \text { for every } \quad \Omega \subset D \quad \text { with } \quad|\Omega| \leq \delta_{0},
$$

where $P(\Omega ; D)=\mathcal{H}^{d-1}\left(\partial^{*} \Omega \cap D\right)$ is the relative perimeter in the sense of De Giorgi.

Now, let $\widetilde{D}_{n}=\left\{x \cdot \nu_{1}>0\right\} \cap\left\{x \cdot \nu_{2}>0\right\}$ be the unbounded domain formed by the intersection of the two hyperplanes $\left\{x \cdot \nu_{1}=0\right\}$ and $\left\{x \cdot \nu_{2}=0\right\}$ forming (an interior) angle $2 \pi / n$. By reflecting any set $\widetilde{\Omega} \subset D_{n} n$ times around $\left\{x \cdot \nu_{1}=0\right\} \cap\left\{x \cdot \nu_{2}=0\right\}$ we get that, for any fixed mass $m>0$, the set $\widetilde{\Omega}_{m}$ that minimizes the perimeter $P\left(\widetilde{\Omega}_{m} ; \tilde{D}_{n}\right)$, among all sets of Lebesgue measure $m$, is the intersection of $\widetilde{D}_{n}$ with the ball $B(\mathrm{~nm})$ centered in zero of measure $\mathrm{nm}$. In particular, we have that

$$
\begin{aligned}
\left|\widetilde{\Omega}_{m}\right|^{(d-1) / d} & =m^{(d-1) / d}=n^{-(d-1) / d}|B(n m)|^{(d-1) / d} \\
& =n^{-(d-1) / d} C_{d} P(B(n m))=n^{1 / d} C_{d} P\left(\widetilde{\Omega}_{m} ; \tilde{D}_{n}\right),
\end{aligned}
$$


where $C_{d}$ is a dimensional constant. For every set $\Omega \subset D$ we now consider the symmetrized set $\Omega^{*}=\widetilde{\Omega}_{m}$, where $m=|\Omega|$. Thus, we have

$$
P(\Omega ; D) \geq C_{D}|\Omega|^{(d-1) / d}=C_{D}\left|\Omega^{*}\right|^{(d-1) / d}=C_{D} n^{1 / d} C_{d} P\left(\Omega^{*} ; \widetilde{D}_{n}\right) .
$$

Fixing $n$ large enough, such that $n^{1 / d} C_{D} C_{d} \geq 1$, we get $P(\Omega ; D) \geq P\left(\Omega^{*} ; \widetilde{D}_{n}\right)$. Now, for every nonnegative function $v \in H^{1}(D)$ we define the symmetrized function $v^{*} \in H^{1}\left(\widetilde{D}_{n}\right)$ obtained through the symmetrization of each level of $v$, that is, $\left\{v^{*}>\right.$ $t\}=\{v>t\}^{*}$ for every $t \geq 0$. Then, we have $\int_{D} v^{2} d x=\int_{\widetilde{D}_{n}}\left|v^{*}\right|^{2} d x$, while for the gradient of $v$ we proceed precisely as in [8, Example 5.3] and using the co-area formula we estimate

$$
\begin{aligned}
\int_{D}|\nabla v|^{2} d x & =\int_{0}^{+\infty}\left(\int_{\{v=t\}}|\nabla v| d \mathcal{H}^{d-1}\right) d t \\
& \geq \int_{0}^{+\infty}\left(\left(\int_{\{v=t\}}|\nabla v|^{-1} d \mathcal{H}^{d-1}\right)^{-1} \mathcal{H}^{d-1}(\{v=t\} \cap D)^{2}\right) d t \\
& =\int_{0}^{+\infty}\left(\left|f^{\prime}(t)\right|^{-1} \mathcal{H}^{d-1}(\{v=t\} \cap D)^{2}\right) d t \\
& \geq \int_{0}^{+\infty}\left(\left|f^{\prime}(t)\right|^{-1} \mathcal{H}^{d-1}\left(\left\{v^{*}=t\right\} \cap \widetilde{D}_{n}\right)^{2}\right) d t \\
& =\int_{0}^{+\infty}\left(\left(\int_{\left\{v^{*}=t\right\}}\left|\nabla v^{*}\right|^{-1} d \mathcal{H}^{d-1}\right)^{-1} \mathcal{H}^{d-1}\left(\left\{v^{*}=t\right\} \cap \widetilde{D}_{n}\right)^{2}\right) d t \\
& =\int_{0}^{+\infty}\left(\int_{\left\{v^{*}=t\right\}}\left|\nabla v^{*}\right| d \mathcal{H}^{1}\right) d t=\int_{\widetilde{D}_{n}}\left|\nabla v^{*}\right|^{2} d x,
\end{aligned}
$$

where $f(t)=|\{v>t\}|=\left|\left\{v^{*}>t\right\}\right|$. Finally, the claim follows by the Faber-Krahn inequality in $\mathbb{R}^{d}$, which gives that there is a dimensional constant $C_{d}$ for which

$$
\int_{\widetilde{D}_{n}}\left|v^{*}\right|^{2} d x \leq \frac{1}{\lambda_{1}\left(B^{*}\right)} \int_{\widetilde{D}_{n}}\left|\nabla v^{*}\right|^{2} d x=C_{d} n^{2 / d}\left|\left\{v^{*}>0\right\}\right|^{2 / d} \int_{\widetilde{D}_{n}}\left|\nabla v^{*}\right|^{2} d x,
$$

where $\lambda_{1}\left(B^{*}\right)$ is the first eigenvalue of the ball $B^{*}$ (of volume $n\left|\left\{v^{*}>0\right\}\right|$ ), obtained by reflecting $n$ times $\left\{v^{*}>0\right\}$ around $\left\{x \cdot \nu_{1}=0\right\} \cap\left\{x \cdot \nu_{2}=0\right\}$.

Theorem 4.5. Let $f \geq 0, f \in L^{2}(D)$, and $g \in L^{2}(D)$. Suppose that $\Omega \subset \mathbb{R}^{d}$ is a solution of the problem (1.3) such that $|\Omega|<1$. Then $\{f g<0\} \subset \Omega$.

Proof. Suppose by contradiction that this is not the case. Then there is a point $x_{0} \in D$ such that $x_{0}$ is a point of density 0 for $\Omega$ and $x_{0}$ is a Lebesgue point for $f$ and $g$ with $f\left(x_{0}\right)>0$ and $g\left(x_{0}\right)<0$; that is,

$$
\begin{aligned}
& \lim _{r \rightarrow 0} \frac{1}{\left|B_{r}\right|} \int_{B_{r}\left(x_{0}\right)} f(x) d x=f\left(x_{0}\right)>0, \\
& \lim _{r \rightarrow 0} \frac{1}{\left|B_{r}\right|} \int_{B_{r}\left(x_{0}\right)} g(x) d x=g\left(x_{0}\right)<0, \\
& \lim _{r \rightarrow 0} \frac{\left|\Omega \cap B_{r}\left(x_{0}\right)\right|}{\left|B_{r}\right|}=0 .
\end{aligned}
$$


Let $r>0$ be fixed. Consider the functions $u, v$ solutions of the problems

$$
\begin{array}{ll}
-\Delta u=f \quad \text { in } \Omega, \quad u \in H_{0}^{1}(\Omega), & \\
-\Delta v=f \quad \text { in } B_{r}\left(x_{0}\right), \quad v=u \quad \text { on } \quad \partial B_{r}\left(x_{0}\right),
\end{array}
$$

set $\nu=\Delta u+f \mathbb{1}_{\{u>0\}}$, and take $r>0$ such that $\nu\left(\partial B_{r}\left(x_{0}\right)\right)=0$. The function $v-u$ is a solution of the PDE

$$
-\Delta(v-u)=\nu+f \mathbb{1}_{B_{r}\left(x_{0}\right) \backslash \Omega} \quad \text { in } \quad B_{r}\left(x_{0}\right), \quad v-u \in H_{0}^{1}\left(B_{r}\left(x_{0}\right)\right),
$$

in the sense that for all $\psi \in H_{0}^{1}\left(B_{r}\left(x_{0}\right)\right)$ we have

$$
\int_{B_{r}\left(x_{0}\right)} \nabla(v-u) \cdot \nabla \psi d x=\int_{B_{r}\left(x_{0}\right) \backslash \Omega} \psi f d x+\int_{B_{r}\left(x_{0}\right)} \psi d \nu
$$

In particular, by the maximum principle, we have that $v-u>0$ on $B_{r}\left(x_{0}\right)$. We now show that

$$
\Delta(v-u)+\nu \mathbb{1}_{B_{r}\left(x_{0}\right)}+f \mathbb{1}_{B_{r}\left(x_{0}\right) \backslash \Omega} \geq 0 \quad \text { in } D,
$$

in the sense of distributions. Let $\phi \in C_{c}^{\infty}(D)$ be a nonnegative function. For every $\varepsilon>0$, consider the function

$$
p_{\varepsilon}(t)= \begin{cases}1 & \text { if } t \geq \varepsilon \\ 0 & \text { if } t \leq 0 \\ t / \varepsilon & \text { if } 0 \leq t \leq \varepsilon\end{cases}
$$

Then $p_{\varepsilon}(v-u) \phi \in H_{0}^{1}\left(B_{r}\left(x_{0}\right)\right)$, and so we have

$\int_{B_{r}\left(x_{0}\right)} \nabla(v-u) \cdot \nabla\left(p_{\varepsilon}(v-u) \phi\right) d x=\int_{B_{r}\left(x_{0}\right) \backslash \Omega} p_{\varepsilon}(v-u) \phi f d x+\int_{B_{r}\left(x_{0}\right)} p_{\varepsilon}(v-u) \phi d \nu$,

which, by developing the gradient, gives

$\int_{B_{r}\left(x_{0}\right)} p_{\varepsilon}(v-u) \nabla(v-u) \cdot \nabla \phi d x \leq \int_{B_{r}\left(x_{0}\right) \backslash \Omega} p_{\varepsilon}(v-u) \phi f d x+\int_{B_{r}\left(x_{0}\right)} p_{\varepsilon}(v-u) \phi d \nu$.

Passing to the limit as $\varepsilon \rightarrow 0$, we obtain

$$
\int_{B_{r}\left(x_{0}\right)} \nabla(v-u) \cdot \nabla \phi d x \leq \int_{B_{r}\left(x_{0}\right) \backslash \Omega} \phi f d x+\int_{B_{r}\left(x_{0}\right)} \phi d \nu
$$

which concludes the proof of (4.3). Define now $\tilde{u} \in H_{0}^{1}(D)$ by

$$
\tilde{u}(x)= \begin{cases}u(x) & \text { if } x \in D \backslash B_{r}\left(x_{0}\right), \\ v(x) & \text { if } x \in B_{r}\left(x_{0}\right) .\end{cases}
$$

We aim to show that $\Delta \tilde{u}+f \mathbb{1}_{\{\tilde{u}>0\}} \geq 0$ on $D$. In fact, using $\phi$ as a test function for 
$\Delta \tilde{u}+f \mathbb{1}_{\{\tilde{u}>0\}}$, we have

$$
\begin{aligned}
\int_{D}(-\nabla \tilde{u} \cdot \nabla \phi+ & \left.f \mathbb{1}_{\{\tilde{u}>0\}} \phi\right) d x \\
= & \int_{\Omega \backslash B_{r}\left(x_{0}\right)}(-\nabla u \cdot \nabla \phi+f \phi) d x+\int_{B_{r}\left(x_{0}\right)}(-\nabla v \cdot \nabla \phi+f \phi) d x \\
& \quad+\int_{\Omega \cap B_{r}\left(x_{0}\right)}(-\nabla u \cdot \nabla \phi+f \phi) d x-\int_{\Omega \cap B_{r}\left(x_{0}\right)}(-\nabla u \cdot \nabla \phi+f \phi) d x \\
= & \int_{\Omega}(-\nabla u \cdot \nabla \phi+f \phi) d x+\int_{B_{r}\left(x_{0}\right)}\left(-\nabla(v-u) \cdot \nabla \phi+f \phi \mathbb{1}_{\left.B_{r}\left(x_{0}\right) \backslash \Omega\right) d x}\right. \\
\geq & \int_{D} \phi d \nu-\int_{B_{r}\left(x_{0}\right)} \phi d \nu \geq 0,
\end{aligned}
$$

which proves the claim. Thus, by Lemma 4.2 , we have that there is a capacitary measure $\tilde{\mu} \in \mathcal{M}_{\text {cap }}(D)$ such that $\Omega_{\tilde{\mu}}=\{\tilde{u}>0\}=\Omega \cup B_{r}\left(x_{0}\right)$ and

$$
-\Delta \tilde{u}+\tilde{\mu} \tilde{u}=f \quad \text { on } D, \quad \tilde{u} \in H_{\tilde{\mu}}^{1}(D) .
$$

Now, by the optimality of $\Omega$ we have that for $r>0$ sufficiently small

$$
0 \leq \int_{D} g \tilde{u} d x-\int_{D} g u d x=\int_{B_{r}\left(x_{0}\right)} g(v-u) d x
$$

In order to conclude, it is now sufficient to study the asymptotic behavior of the integral on the right-hand side as $r \rightarrow 0$. Assume for simplicity that $x_{0}=0$. We consider the functions $w$ and $h$ solutions of the equations

$$
\begin{aligned}
& -\Delta w=f \quad \text { in } B_{r}, \quad w \in H_{0}^{1}\left(B_{r}\right), \\
& \Delta h=0 \quad \text { in } B_{r}, \quad h-u \in H_{0}^{1}\left(B_{r}\right),
\end{aligned}
$$

and we set

$f_{r}(x)=f(r x), g_{r}(x)=g(r x), w_{r}(x)=\frac{1}{r^{2}} w(r x), h_{r}(x)=\frac{1}{r^{2}} h(r x), u_{r}(x)=\frac{1}{r^{2}} u(r x)$.

We notice the following:

(i) Since $x_{0}=0$ is a Lebesgue point for both $f$ and $g$, we have that $f_{r} \rightarrow f(0)$ and $g_{r} \rightarrow g(0)$ strongly in $L^{2}\left(B_{1}\right)$, as $r \rightarrow 0$.

(ii) The function $w_{r}$ is a solution of the equation

$$
-\Delta w_{r}=f_{r} \quad \text { in } B_{1}, \quad w \in H_{0}^{1}\left(B_{1}\right),
$$

and $w_{r} \rightarrow w_{0}$ strongly in $H_{0}^{1}\left(B_{1}\right)$, where $w_{0}(x)=\frac{f(0)}{2 d}\left(1-|x|^{2}\right)$ is the solution of

$$
-\Delta w_{0}=f(0) \quad \text { in } B_{1}, \quad w_{0} \in H_{0}^{1}\left(B_{1}\right) .
$$

(iii) There is a constant $C$, not depending on $r$, such that

$$
\int_{B_{1}}\left|\nabla h_{r}\right|^{2} d x \leq \int_{B_{1}}\left|\nabla u_{r}\right|^{2} d x \leq C .
$$

The first inequality is due to the harmonicity of $h_{r}$, while the second one is a consequence of Lemma 4.3. Thus, $\left\|h_{r}-u_{r}\right\|_{H^{1}}^{2} \leq C$, and so, up to a subsequence, 
we may assume that $z_{r}=h_{r}-u_{r}$ converges weakly in $H_{0}^{1}\left(B_{1}\right)$ and strongly in $L^{2}\left(B_{1}\right)$ to some function $z_{0} \in H_{0}^{1}\left(B_{1}\right)$. We now prove that $z_{0}=0$. In fact, given a function $\phi \in C_{c}^{\infty}\left(B_{1}\right)$ we have that

$$
\begin{aligned}
\int_{B_{1}} \nabla \phi \cdot \nabla z_{r} d x & =-\int_{B_{1}} \nabla \phi \cdot \nabla u_{r} d x \\
& \leq\|\nabla \phi\|_{L^{\infty}}\left|\left\{u_{r} \neq 0\right\} \cap B_{1}\right|^{1 / 2}\left(\int_{B_{1}}\left|\nabla u_{r}\right|^{2} d x\right)^{1 / 2} \\
& \leq C\|\nabla \phi\|_{L^{\infty}}\left(\frac{\left|\Omega \cap B_{r}\right|}{\left|B_{r}\right|}\right)^{1 / 2}
\end{aligned}
$$

where the equality is due to the fact that $h_{r}$ is harmonic, the first inequality is by Cauchy-Schwarz, and the last inequality is due to the estimate (4.5). Now since the density of $\Omega$ is zero in 0 , passing to the limit as $r \rightarrow 0$, we obtain

$$
\int_{B_{1}} \nabla \phi \cdot \nabla z_{0} d x \leq 0
$$

Since $\phi$ is arbitrary we obtain that $z_{0}$ is harmonic in $B_{1}$, and since $z_{0}=0$ on $\partial B_{1}$ we get that $z_{0}=0$. Thus we conclude that

$$
\lim _{r \rightarrow 0} \int_{B_{1}}\left|h_{r}-u_{r}\right|^{2} d x=0 .
$$

By the results from (i), (ii), and (iii), we get that

$$
\int_{B_{r}} g(v-u) d x=r^{2-d} \int_{B_{1}} g_{r}\left(w_{r}+h_{r}-u_{r}\right) d x=r^{2-d}\left(\int_{B_{1}} g\left(x_{0}\right) w_{0}(x) d x+o(r)\right),
$$

which is strictly negative, for $r>0$ sufficiently small, and so contradicts (4.4).

Remark 4.6. Since the resolvent operator is self-adjoint, in Theorem 4.5 we may equivalently assume $g \geq 0$ and deduce that if $|\Omega|<1$ then $\{g f<0\} \subset \Omega$. By a simple change of sign in the data we also have that if $f \leq 0$ (or if $g \leq 0$ ) and $|\Omega|<1$, then $\{g f<0\} \subset \Omega$.

5. Unconstrained minimizers and the obstacle problem. Let $D \subset \mathbb{R}^{d}$ be a bounded open set. We say that $\Omega \subset D$ is an unconstrained minimizer if it is a solution of the optimization problem

$$
\min \left\{\int_{\Omega} R_{\Omega}(g) f(x) d x: \Omega \text { quasi open, } \Omega \subset D\right\},
$$

where we removed the measure constraint on $\Omega$. In Proposition 5.4 we prove that the solution of (5.1) is related to the solution of the obstacle problem

$$
\min \left\{\frac{1}{2} \int_{D}|\nabla v|^{2} d x+\int_{D} g(x) v(x) d x: v \in H_{0}^{1}(D), v \geq 0 \text { on } D\right\} .
$$

We first prove the following lemma characterizing the solutions of (5.2).

Lemma 5.1. Let $D \subset \mathbb{R}^{d}$ be a bounded open set and $g \in L^{2}(D)$. Then the solution $v$ of the obstacle problem (5.2) satisfies

$$
v=\sup _{\Omega \subset D} v_{\Omega},
$$


where the maximum is over all quasi open subsets $\Omega \subset D$ and $v_{\Omega}$ is the solution of

$$
\Delta v_{\Omega}=g \quad \text { in } \Omega, \quad v_{\Omega} \in H_{0}^{1}(\Omega) .
$$

Proof. Suppose that $\Omega \subset D$ is a quasi open set. It is sufficient to prove that $v \geq v_{\Omega}$ in $D$. Indeed, set

$$
J(u)=\frac{1}{2} \int_{D}|\nabla u|^{2} d x+\int_{D} u(x) g(x) d x,
$$

and consider the test functions $v \vee v_{\Omega}$ and $v \wedge v_{\Omega}$. Since $v \vee v_{\Omega} \leq 0$ in $D$ and $v \wedge v_{\Omega} \in H_{0}^{1}(\Omega)$, we have the inequalities

$$
J(v) \leq J\left(v \vee v_{\Omega}\right) \quad \text { and } \quad J\left(v_{\Omega}\right) \leq J\left(v \wedge v_{\Omega}\right) .
$$

On the other hand, by the definition of $J$ we have

$$
J(v)+J\left(v_{\Omega}\right)=J\left(v \vee v_{\Omega}\right)+J\left(v \wedge v_{\Omega}\right) .
$$

Thus, we obtain

$$
J(v)=J\left(v \vee v_{\Omega}\right) \quad \text { and } \quad J\left(v_{\Omega}\right)=J\left(v \wedge v_{\Omega}\right) .
$$

By the uniqueness of the solution of the obstacle problem and of the equation (5.4), we have that $v=v \vee v_{\Omega}$ and $v_{\Omega}=v \wedge v_{\Omega}$, which concludes the proof.

Remark 5.2. The supremum in (5.3) is realized by the quasi open set $\Omega=\{v>0\}$.

Remark 5.3. By the density of the (smooth) open sets in the family of quasi open sets we have that

$$
v=\sup \left\{v_{\Omega}: \Omega \text { open, } \Omega \subset D\right\} .
$$

Proposition 5.4. Let $D \subset \mathbb{R}^{d}$ be a bounded open set, and let $f, g \in L^{2}(D)$ with $f \geq 0$ on $D$. Then the unique minimizer of the unconstrained problem (5.1) is the quasi open set $\Omega=\{v>0\}$, where $v$ is the solution of the obstacle problem (5.2).

Proof. Let $\Omega \subset D$ be a quasi open set. By Lemma 5.1 we have that $v \geq v_{\Omega}$. Since $f \geq 0$ we have that

$$
\int_{D} R_{\Omega}(g) f d x=-\int_{D} v_{\Omega} f d x \geq-\int_{D} v f d x=\int_{D} R_{\{v>0\}}(g) f d x
$$

which concludes the proof.

As a corollary we obtain the following result.

Corollary 5.5. Let $D \subset \mathbb{R}^{d}$ be a bounded open set, and let $f, g \in L^{2}(D)$ with $f \geq 0$ in D. Suppose that $\Omega \subset D$ is a solution of the optimization problem (1.3) such that $|\Omega|<1$. Then the following hold:

(i) if $g \in L^{p}(D)$, for some $p>d$, then $\Omega$ is an open subset of $D$ and the function $v=R_{\Omega}(g)$ is $C^{1, \beta}$ regular in $D$, where $\beta=1-d / p$;

(ii) if the set $\{g>0\}$ is open and $g:\{g>0\} \rightarrow \mathbb{R}_{+}$is Hölder continuous, then $v$ is $C^{1,1}$ regular in the set $\{g>0\}$ and $|\nabla v|=0$ on the free boundary $\partial \Omega \cap\{g>0\}$;

(iii) under the hypotheses from the previous point, the free boundary $\partial \Omega \cap\{g>0\}$ can be decomposed into two disjoint sets Reg $(\partial \Omega)$ and Sing $(\partial \Omega)$, where

- $\operatorname{Reg}(\partial \Omega)$ is an open subset of $\partial \Omega \cap\{g>0\}$ and is locally the graph of a $C^{1, \alpha}$ function, for some $\alpha>0$; if $g \in C^{\infty}(\{g>0\})$, then $\operatorname{Reg}(\partial \Omega)$ is smooth; 
- $\operatorname{Sing}(\partial \Omega)=\bigcup_{k=0}^{d-1} S_{k}$, where each $S_{k}$ is contained in the union of countably many submanifolds of dimension $k$ and class $C^{1, \log }\left(C^{1, \alpha}\right.$ in dimension two).

Proof. We first notice that since $\Omega$ is such that $|\Omega|<1$, it is an unconstrained minimizer of (5.1) in the set $\tilde{D}=\Omega \cup B_{r}\left(x_{0}\right) \cap D$, for every sufficiently small ball $B_{r}\left(x_{0}\right)$. By Proposition 5.4, the function $R_{\Omega}(-g)$ is a solution of the obstacle problem $(5.2)$ in $\tilde{D}$. Thus, all the regularity results for the obstacle problem are valid for $v=R_{\Omega}(g)$, particularly the statements (i), (ii), and (iii). For the proof of (i) we refer the reader to [3]; (ii) was first proved in [9] (see also [15] for the higher regularity of the free boundary and [17] for an alternative approach); for the proof of (iii) we refer the reader to the recent papers [10] and [13].

6. The case of radially symmetric cost functionals. In this section we consider a special class of functionals, where $f=1$ and $g: \mathbb{R}^{d} \rightarrow \mathbb{R}$ is radially symmetric and nondecreasing on each radius. It is natural to conjecture that in this situation the optimal set is a ball centered at the origin. In the case when $g \leq 0$ this follows by a classical symmetrization argument; on the other hand, if $g$ changes sign, the cost functional is nonmonotone and the known symmetrization results fail in the comparison argument of a general domain with a ball of the same measure. In this section we prove the following proposition.

Proposition 6.1. Suppose that $f=1$ and $g: \mathbb{R}^{d} \rightarrow \mathbb{R}$ is a given radially symmetric nondecreasing function such that $g(0)<0$. Then, setting

$$
R_{g}=\sup \left\{R>0: \int_{0}^{R} r^{d-1} g(r) d r \leq 0\right\},
$$

the ball, of radius $\inf \left\{\omega_{d}^{-1 / d}, R_{g}\right\}$ and centered at the origin, is a solution of the problem

$$
\min \left\{\int_{D} g(x) u_{\Omega}(x) d x: \Omega \subset \mathbb{R}^{d}, \Omega \text { quasi open, }|\Omega| \leq 1\right\} .
$$

Remark 6.2. The condition $g(0)<0$ ensures that the solution of $(6.1)$ is nontrivial. Indeed, if $g \geq 0$ on $\mathbb{R}^{d}$, then the empty set is a solution as well as every quasi open subset of $\{g=0\}$.

As a consequence of Proposition 6.1 we obtain the following example.

Example 6.3. Suppose that $f=1$ and $g=-\mathbb{1}_{B_{r_{0}}}+\mathbb{1}_{B_{r_{0}}^{c}}$ for some radius $r_{0}>0$. Then the solution $\Omega_{\text {opt }}$ of the problem (6.1) is unique and is given by the ball of volume $\min \left\{2\left|B_{r_{0}}\right|, 1\right\}$. Indeed, the solution is a ball $B_{R}$ that contains the set $B_{r_{0}}=\{g<0\}$. The energy of the ball $B_{R}$ is given by the formula

$$
f(R)=d \omega_{d}\left[-\int_{0}^{r_{0}} \frac{R^{2}-r^{2}}{2 d} r^{d-1} d r+\int_{r_{0}}^{R} \frac{R^{2}-r^{2}}{2 d} r^{d-1} d r\right] .
$$

Taking the derivative with respect to $R$, we get that

$$
f^{\prime}(R)=\frac{\omega_{d}}{d} R\left(R^{d}-2 r_{0}^{d}\right) .
$$

Thus, the function $f$ achieves its minimum at $2^{1 / d} r_{0}$, if $2 r_{0}^{d} \leq \omega_{d}^{-1 / d}$, and at 1 , if $2 r_{0}^{d} \geq \omega_{d}^{-1 / d}$, which gives the claim.

The rest of this section is dedicated to the proof of Proposition 6.1. 
6.1. The Schwarz rearrangement of a torsion function. Let $\Omega \subset \mathbb{R}^{d}$ be a bounded open or quasi open set and $u \in H_{0}^{1}(\Omega)$ be the torsion function of $\Omega$, that is, the solution of

$$
-\Delta u=1 \quad \text { in } \Omega, \quad u \in H_{0}^{1}(\Omega) .
$$

Let $\Omega^{*}$ be the ball centered at zero of measure $|\Omega|$, and let $u^{*}: \Omega^{*} \rightarrow \mathbb{R}$ be the radially decreasing rearrangement of $u$. We set $M=\|u\|_{L^{\infty}(\Omega)}$ and $\Omega_{t}=\{u>t\}$ for every $t \in[0, M]$. Then the set $\Omega_{t}^{*}=\left\{u^{*}>t\right\}$ is the ball centered at zero of measure $\left|\Omega_{t}^{*}\right|=\left|\Omega_{t}\right|$. On every set $\Omega_{t}^{*}$ we consider the function $w_{t}$ solution of the PDE

$$
-\Delta w_{t}=1 \quad \text { in } \Omega_{t}^{*}, \quad w_{t} \in H_{0}^{1}\left(\Omega_{t}^{*}\right) .
$$

A well-known result of Talenti [16] gives that

$$
u^{*}(x)-t \leq w_{t}(x) \text { for every } x \in \Omega_{t}^{*} \text { and every } t \in[0, M] .
$$

In the next lemma we use this comparison to obtain that the function $u^{*}$ is itself a solution of a certain PDE on $\Omega^{*}$.

Lemma 6.4. Let $\Omega \subset \mathbb{R}^{d}$ be a bounded quasi open set, and let $u$ be the solution of (6.2). Then the Steiner symmetrization $u^{*}$ of $u$ is a solution of the equation

$$
-\operatorname{div}\left(\left(1+a\left(u^{*}\right)\right) \nabla u^{*}\right)=1 \quad \text { in } \Omega^{*}, \quad u^{*} \in H_{0}^{1}\left(\Omega^{*}\right),
$$

where $a:[0, M] \rightarrow \mathbb{R}$ is a nonnegative function.

Proof. We use the notation introduced at the beginning of the section. Let $f$ : $[0, M] \rightarrow \mathbb{R}$ be a given $C^{1}$ function such that $f(0)=0$. Then $f\left(u^{*}\right) \in H_{0}^{1}\left(\Omega^{*}\right)$, and we have, by using the co-area formula,

$$
\begin{aligned}
\int_{\Omega^{*}} \nabla u^{*} \cdot \nabla f\left(u^{*}\right) d x-\int_{\Omega^{*}} f\left(u^{*}\right) d x= & \int_{\Omega^{*}} f^{\prime}\left(u^{*}\right)\left|\nabla u^{*}\right|^{2} d x-\int_{\Omega} f(u) d x \\
= & \int_{0}^{M} f^{\prime}(t) \int_{\partial \Omega_{t}^{*}}\left|\nabla u^{*}\right| d \mathcal{H}^{d-1} d t-\int_{\Omega} f(u) d x \\
= & -\int_{0}^{M} f^{\prime}(t) a(t) \int_{\partial \Omega_{t}^{*}}\left|\nabla u^{*}\right| d \mathcal{H}^{d-1} d t \\
& +\int_{0}^{M} f^{\prime}(t) \int_{\partial \Omega_{t}^{*}}\left|\nabla w_{t}\right| d \mathcal{H}^{d-1} d t-\int_{\Omega} f(u) d x,
\end{aligned}
$$

where we set

$$
a(t):=\frac{\int_{\partial \Omega_{t}^{*}}\left|\nabla w_{t}\right| d \mathcal{H}^{d-1}-\int_{\partial \Omega_{t}^{*}}\left|\nabla u^{*}\right| d \mathcal{H}^{d-1}}{\int_{\partial \Omega_{t}^{*}}\left|\nabla u^{*}\right| d \mathcal{H}^{d-1}} .
$$

By the co-area formula the function $a$ is well defined for a.e. $t \in[0, M]$, and, thanks to (6.3), it turns out to be nonnegative. We also notice that the difference of the last two terms in (6.5) vanishes. Indeed, using an integration by parts for $w_{t}$ we get

$$
\int_{\partial \Omega_{t}^{*}}\left|\nabla w_{t}\right| d \mathcal{H}^{d-1}=-\int_{\partial \Omega_{t}^{*}} \frac{\partial w_{t}}{\partial n} d \mathcal{H}^{d-1}=-\int_{\Omega_{t}^{*}} \Delta w_{t} d x=\left|\Omega_{t}^{*}\right| .
$$


Analogously, since $u-t$ is the solution of $-\Delta(u-t)=1$ on $\Omega_{t}$, we get

$$
\int_{\partial \Omega_{t}}|\nabla u| d \mathcal{H}^{d-1}=-\int_{\partial \Omega_{t}} \frac{\partial u}{\partial n} d \mathcal{H}^{d-1}=-\int_{\Omega_{t}} \Delta u d x=\left|\Omega_{t}\right| .
$$

Since $\left|\Omega_{t}\right|=\left|\Omega_{t}^{*}\right|$, we obtain

$$
\begin{aligned}
\int_{0}^{M} f^{\prime}(t) \int_{\partial \Omega_{t}^{*}}\left|\nabla w_{t}\right| d \mathcal{H}^{d-1} d t & =\int_{0}^{M} f^{\prime}(t) \int_{\partial \Omega_{t}}|\nabla u| d \mathcal{H}^{d-1} d t \\
& =\int_{\Omega_{t}} f^{\prime}(u)|\nabla u|^{2} d x=\int_{\Omega} f(u) d x
\end{aligned}
$$

On the other hand, by the co-area formula, the first term in the last line of (6.5) can be rewritten as

$$
\begin{aligned}
\int_{0}^{M} f^{\prime}(t) a(t) \int_{\partial \Omega_{t}^{*}}\left|\nabla u^{*}\right| d \mathcal{H}^{d-1} d t & =\int_{\Omega^{*}} f^{\prime}\left(u^{*}\right) a\left(u^{*}\right)\left|\nabla u^{*}\right|^{2} d x \\
& =\int_{\Omega^{*}} a\left(u^{*}\right) \nabla u^{*} \cdot \nabla f\left(u^{*}\right) d x .
\end{aligned}
$$

Thus, by (6.5) we infer

$$
\int_{\Omega^{*}}\left(1+a\left(u^{*}\right)\right) \nabla u^{*} \cdot \nabla f\left(u^{*}\right) d x=\int_{\Omega^{*}} f\left(u^{*}\right) d x .
$$

Since the equality is true for every $f$, with $f(0)=0$, we obtain (6.4).

In the next subsection we establish which is the optimal function $a$ on a ball of fixed radius $R$.

6.2. An optimization problem for radially decreasing functions. Let $a$ : $\left[0, R_{0}\right] \rightarrow[0,+\infty)$ be a given nonnegative measurable function. Let $R \leq R_{0}$ and $u_{a, R}$ be the solution of the PDE

$$
-\operatorname{div}((1+a) \nabla u)=1 \quad \text { in } B_{R}, \quad u \in H_{0}^{1}\left(B_{R}\right) .
$$

Then $u_{a, R}=u_{a, R}(r)$ is radially symmetric and is a solution of the problem

$$
-\frac{1}{r^{d-1}} \partial_{r}\left(r^{d-1}(1+a(r)) \partial_{r} u(r)\right)=1 \quad \text { in }(0, R), \quad u(R)=u^{\prime}(0)=0 .
$$

Integrating in $r$ we get that $u_{a, R}$ is explicitly given by

$$
u_{a, R}(r)=\frac{1}{d} \int_{r}^{R} \frac{s}{1+a(s)} d s .
$$

We consider a radial nondecreasing function $g: \mathbb{R}^{d} \rightarrow \mathbb{R}$ such that $g(0)<0$ and the associated cost functional $\mathcal{F}(a, R)$ given by

$$
\mathcal{F}(a, R)=\int_{B_{R}} g(x) u_{a, R}(x) d x .
$$

Setting

$$
G(s)=\int_{0}^{s} r^{d-1} g(r) d r,
$$


we obtain

$$
\mathcal{F}(a, R)=\frac{1}{d} \int_{0}^{R} r^{d-1} g(r) \int_{r}^{R} \frac{s}{1+a(s)} d s d r=\frac{1}{d} \int_{0}^{R} \frac{G(s) s}{1+a(s)} d s .
$$

Since $g$ is nondecreasing and $g(0)<0$, we have that the set $\{g \leq 0\}$ is an interval of the form $\left[0, R_{g}\right]$ (we set $R_{g}=+\infty$ in the case when $g \leq 0$ on $\mathbb{R}^{d}$ ). Then we have

$$
\begin{cases}\mathcal{F}(a, R) \geq \mathcal{F}(0, R) & \text { if } R \leq R_{g}, \\ \mathcal{F}(a, R) \geq \mathcal{F}\left(0, R_{g}\right) & \text { if } R>R_{g} .\end{cases}
$$

Indeed, if $R \leq R_{g}$, then $G \leq 0$ and (6.6) follows since in this case the functional $\mathcal{F}(a, R)$ is monotone increasing in $a$. On the other hand, if $R>R_{g}$, we have that

$$
\mathcal{F}(a, R)=\frac{1}{d} \int_{0}^{R} \frac{G(s) s}{1+a(s)} d s \geq \frac{1}{d} \int_{0}^{R_{g}} \frac{G(s) s}{1+a(s)} d s=\mathcal{F}\left(a, R_{g}\right),
$$

and (6.6) again follows since $\mathcal{F}\left(a, R_{g}\right)$ is monotone increasing in $a$.

Proof of Proposition 6.1. Given a quasi open set $\Omega \subset \mathbb{R}^{d}$ and a function $u$ solution of (6.2), we consider the ball $\Omega^{*}$ of measure $\Omega$ and the symmetrized function $u^{*}$. By the Riesz inequality we have that

$$
\int_{\Omega} g(x) u(x) d x \geq \int_{\Omega^{*}} g^{*}(x) u^{*}(x) d x
$$

By Lemma 6.4 we get that

$$
\int_{\Omega^{*}} g^{*}(x) u^{*}(x) d x=\mathcal{F}\left(a\left(u^{*}\right), R\right),
$$

where $R$ is the radius of $\Omega^{*}$. Now the inequality (6.6) gives that

$$
\mathcal{F}\left(a\left(u^{*}\right), R\right) \geq \mathcal{F}\left(0, R \wedge R_{g}\right) \geq \mathcal{F}\left(0, \omega_{d}^{-1 / d} \wedge R_{g}\right) .
$$

If $B$ is the ball of radius $\omega_{d}^{-1 / d} \wedge R_{g}$, by the definition of $\mathcal{F}$ we have that

$$
\mathcal{F}\left(0, R \wedge R_{g}\right)=\int_{B} g(x) u_{B}(x) d x
$$

which concludes the proof of Proposition 6.1.

\section{REFERENCES}

[1] G. Allaire And C. Dapogny, A deterministic approximation method in shape optimization under random uncertainties, SMAI J. Comput. Math., 1 (2015), pp. 83-143.

[2] J. C. Bellido, G. Buttazzo, and B. Velichkov, Worst-case shape optimization for the Dirichlet energy, Nonlinear Anal., 153 (2017), pp. 117-129.

[3] H. Brezis and G. Stampacchia, Sur la régularité de la solution d'inéquations elliptiques, Bull. Soc. Math. France, 96 (1968), pp. 153-180.

[4] D. Bucur and G. Buttazzo, Variational Methods in Shape Optimization Problems, Progr. Nonlinear Differential Equations Appl. 65, Birkhäuser Boston, Boston, 2005.

[5] G. Buttazzo And G. Dal Maso, Shape optimization for Dirichlet problems: Relaxed solutions and optimality conditions, Appl. Math. Optim., 23 (1991), pp. 17-49. 
[6] G. Buttazzo and G. Dal Maso, An existence result for a class of shape optimization problems, Arch. Ration. Mech. Anal., 122 (1993), pp. 183-195.

[7] G. Buttazzo, A. Gerolin, B. Ruffini, and B. Velichkov, Optimal potentials for Schrödinger operators, J. Éc. Polytech. Math., 1 (2014), pp. 71-100.

[8] G. Buttazzo and B. Velichkov, The spectral drop problem, in Recent Advances in Partial Differential Equations and Applications, Contemp. Math. 666, AMS, Providence, RI, 2016, pp. 111-135.

[9] L. A. Caffarelli, The obstacle problem revisited, J. Fourier Anal. Appl., 4 (1998), pp. 383-402.

[10] M. Colombo, L. Spolaor, and B. Velichkov, A logarithmic epiperimetric inequality for the obstacle problem, Geom. Funct. Anal., to appear; preprint, https://arxiv.org/abs/1708. $02045,2017$.

[11] G. Dal Maso and U. Mosco, Wiener's criterion and $\Gamma$-convergence, Appl. Math. Optim., 15 (1987), pp. 15-63.

[12] G. Dal Maso and F. Murat, Asymptotic behaviour and correctors for Dirichlet problems in perforated domains with homogeneous monotone operators, Ann. Scuola Norm. Sup. Pisa Cl. Sci., 24 (1997), pp. 239-290.

[13] A. Figalli and J. Serra, On the Fine Structure of the Free Boundary for the Classical Obstacle Problem, preprint, https://arxiv.org/abs/1709.04002, 2017.

[14] A. Henrot and M. Pierre, Variation et Optimisation de Formes. Une Analyse Géométrique, Math. Appl. (Berlin) 48, Springer-Verlag, Berlin, 2005.

[15] D. Kinderlehrer and L. NirenberG, Regularity in free boundary problems, Ann. Scuola Norm. Sup. Pisa Cl. Sci. (4), 4 (1977), pp. 373-391.

[16] G. TAlenti, Estimates for eigenvalues of Sturm-Liouville problems, in General Inequalities, 4 (Oberwolfach, 1983), Internat. Ser. Numer. Math. 71, Birkhäuser Verlag, Basel, 1984, pp. 341-350.

[17] G. S. WeIss, A homogeneity improvement approach to the obstacle problem, Invent. Math., 138 (1999), pp. 23-50. 